\title{
CAPACIDADES EMPRENDEDORAS Y PERSONALIDAD EFICAZ EN ESTUDIANTES DE UNA UNIVERSIDAD PRIVADA DE LIMA
}

\author{
ENTREPRENEUR CAPACITIES AND EFFECTIVE PERSONALITY IN STUDENTS OF A \\ PRIVATE UNIVERSITY OF LIMA
}

\author{
Manuel Torres Valladares*, Manuel Torres Lajo \\ Universidad Nacional Mayor de San Marcos \\ RECIBIDO 15-8-2013, ACEPTADO: 12-11-2013)
}

\begin{abstract}
RESUMEN
Uno de los grandes problemas que padece nuestro país es que en el mundo universitario los jóvenes son preparados para ser empleados y no para ser empresarios, para recibir órdenes y no para darlas, para usar herramientas y técnicas ya creadas y no para crearlas o recrearlas. Esto ha llevado a una realidad social en la cual no se incentiva ni premia la inventiva y la creatividad, no se valora la rápida resolución de problemas y mucho menos la gestión innovadora. Es por eso que el propósito básico de la presente investigación, consiste en tener una descripción de las características de la personalidad eficaz de los estudiantes y como estas se relacionan con sus capacidades emprendedoras.
\end{abstract}

En el desarrollo de la presente investigación se utilizó el Inventario de Personalidad eficaz de Del Buey, M. y el inventario de Capacidades emprendedoras de Moriano, J. Los análisis estadísticos a las que fueron sometidas las pruebas nos indican que los instrumentos son válidos y confiables. Estas pruebas fueron aplicadas a 155 alumnos de la Facultad de Administración de una Universidad privada de Lima.

Los resultados estadísticos nos indican que la personalidad eficaz de los estudiantes se encuentra relacionada con sus capacidades emprendedoras, así mismo se encontró que los estudiantes presentan diferencias significativas considerando su ciclo académico, en la variable personalidad eficaz.

Palabras clave: Personalidad eficaz, capacidades emprendedoras, liderazgo, emprededorismo.

\section{ABSTRACT}

One of the biggest problems that our country faces is that in the university world, young people are trained to be employees and not to be entrepreneurs, to recieve orders and not to give them, to use tools and techniques already created and not to create or recreate them. This has lead into a social reality in which inventiviness and creativity are not incetivated

\footnotetext{
* Docente Principal de la Facultad de Psicología UNMSM. E-mail: manueltorres67@yahoo.es
} 
nor rewarded, the quick solving of problems is not appreciated and much less, innovative managament. For this reason, the main purpose of the current investigation consists in having a description of the characteristics of the students' effective personality and how these relate to their entrepreneur capactities.

In the development of the current investigation were used the Effective Personality Inventory of Del Buey and the Entrepreneur Capacities Inventory of Moriano, J. The statistical analyses the tests were submitted to indicate us the instruments are valid and reliable. These tests were applied to 155 students of the Administration Faculty of a private university of Lima.

The statistical results indicate us that the effective personality of the students is related to their entrepreneur capacities. Moreover, it was found that the students present significant differences regarding to their academic cycle in the variable of effective personality.

Key words: effective personality, entrepreneur capacities, leadership, entrepreneurship.

\section{INTRODUCCIÓN}

Se ha dicho que la formación del hombre en el siglo XXI se encuentra ante el principal reto de su historia: entrenar a las personas para vivir en un mundo cambiante y presidido por la incertidumbre, en el que los individuos han de definir su proyecto vital y estar preparados para habitar un mundo en el que tienen que ser sus protagonistas.

Cada día nos enfrentamos a un mundo desafiante, cuyos problemas exigen personas capaces de afrontar nuevos retos, emprendedores que, más allá de adaptarse, sean capaces de transformar su entorno favorablemente. Es necesario desarrollar una nueva visión, reflexiva, capaz de generar nuevos pensamientos, de saber quiénes somos y a dónde vamos, de valorar el mundo y todo lo que conlleva su transformación en un lugar más justo, productivo y solidario.

Estas capacidades que se necesitan deben ser fomentadas y desarrolladas a través de una didáctica innovadora y creativa basada en una educación emprendedora, educación para el trabajo y educación financiera, que fomente jóvenes independientes, generadores de riqueza en todos los sentidos, dispuestos a asumir riesgos, con motivación al logro, preparados para enfrentar los desafíos actuales, preparados para tener una vida plena. Todas estas cualidades y actitudes son necesarias para el diseño y desarrollo de cualquier proyecto social, educativo, profesional o económico y, en general, en cualquier ámbito o situación de la vida.

La construcción de una cultura emprendedora y solidaria es uno de los fines más ambiciosos y a la vez más necesarios de la sociedad y del sistema educativo actual en tanto la capacidad emprendedora constituye la habilidad de la persona para transformar las ideas en actos. Está relacionada con la creatividad, la innovación, la asunción de riesgos, el trabajo en equipo, autoestima, habilidades de comunicación, planificación y toma de decisiones. Estas cualidades son importantes a considerar en tanto forman parte de los procesos de aprendizaje que la persona va adquiriendo a través de su experiencia vital, por lo que son susceptibles de enseñarse y aprenderse. 
Mencionemos por ejemplo, que a los niños se les enseña a estudiar o jugar en grupo, respetando determinadas reglas. A partir de ello los niños van interiorizando dos capacidades muy importantes para las personas emprendedoras; trabajo en equipo y asunción de compromisos. Es necesario señalar que en edades adultas con una formación adecuada, entrenando y fomentando determinadas estrategias o habilidades, también se pueden desarrollar estas capacidades emprendedoras.

Desde este punto de vista es necesario insistir en la necesidad de que los emprendedores cuenten con cualidades personales y con conocimientos profesionales determinados y si no los tiene debe ser capaz de incorporar a personas que si los tengan no es que los conocimientos profesionales (experiencia en el sector, conocimientos comerciales, técnicos, etc.) sean imprescindibles, pero la persona emprendedora debería tenerlos o saber que necesita apoyo de otras personas desde el inicio de la actividad emprendedora.

Iniciar una actividad empresarial implica aspectos positivos y negativos a considerar. Por ejemplo hay dinero que se arriesga también mucho tiempo de dedicación al trabajo y a veces las exigencias de la empresa pueden llegar a desbordarlo. Es por eso que resulta conveniente que cuente con un entorno que le apoye, así como con unos recursos económicos suficientes para mantenerse hasta que el negocio se ponga en funcionamiento y produzca los suficientes beneficios.

En este proceso la Universidad tiene un importante papel que cumplir, en tanto su misión no debe ser solo formar empleados sino emprendedores, en tanto el problema del desempleo se complica no solo por la crisis económica sino también por la deficiente educación que no corresponde a la época de exigencias y competencias tecnológicas, esto se debe revertir en el corto plazo. De esta manera es de suponer que cuando una persona se prepara a nivel universitario aspira a emplearse o ser generador de su propio empleo; sin embargo todo parece indicar que en los egresados existen dificultades para que estos sean autogestores de su propio desarrollo laboral, lo que evidencia una problemática a nivel de emprendimiento.

En una necesidad el implantar el emprendimiento como opción de progreso, los estudios en su mayor parte se han centrado en estudiar las empresas y no al empresario como propiciador o creador de las mismas. Es por eso que las nuevas investigaciones deben centrarse más en los estudios sobre el empresario y sobre el espíritu emprendedor y en ellas deben participar todas las ciencias.

Como consecuencia de lo expuesto, los problemas de la presente investigación se plantean de la siguiente manera:

$>$ ¿Qué relación existe entre la personalidad eficaz y la capacidad emprendedora en los estudiantes de la Facultad de Administración de una Universidad privada de Lima?

¿Qué relaciones existen entre los diferentes componentes de la personalidad eficaz y las diversas capacidades emprendedoras, en los estudiantes de la Facultad de Administración de una Universidad privada de Lima? 
CAPACIDADES EMPRENDEDORAS Y PERSONALIDAD EFICAZ EN ESTUDIANTES DE UNA UNIVERSIDAD PRIVADA DE LIMA

$>$ ¿Qué diferencias existen entre los alumnos varones y mujeres de la Facultad de Administración de una Universidad privada de Lima respecto de su personalidad eficaz?

$>$ ¿Qué diferencias existen entre los alumnos varones y mujeres de la Facultad de Administración de una Universidad privada de Lima respecto de sus capacidades emprendedoras?

$>$ ¿Qué diferencias existen entre los estudiantes considerando su ciclo académico, de la Facultad de Administración de una Universidad privada de Lima respecto de sus capacidades emprendedoras?

$>$ ¿Qué diferencias existen entre los estudiantes considerando su ciclo académico, de la Facultad de Administración de una Universidad privada de Lima respecto de su personalidad eficaz?

\section{Objetivos}

1. Establecer la relación que existe entre la personalidad eficaz y la capacidad emprendedora en los estudiantes de la Facultad de Administración de una Universidad privada de Lima.

2. Determinar las relaciones que existen entre los diferentes componentes de la personalidad eficaz y las diversas capacidades emprendedoras, en los estudiantes de la Facultad de Administración de una Universidad privada de Lima.

3. Establecer las diferencias que existen entre los alumnos varones y mujeres de la Facultad de Administración de una Universidad privada de Lima respecto de su personalidad eficaz.

4. Establecer las diferencias que existen entre los alumnos varones y mujeres de la Facultad de Administración de una Universidad privada de Lima respecto de sus capacidades emprendedoras.

5. Establecer las diferencias que existen entre los estudiantes considerando su ciclo académico, de la Facultad de Administración de una Universidad privada de Lima respecto de sus capacidades emprendedoras.

6. Establecer las diferencias que existen entre los estudiantes considerando su ciclo académico, de la Facultad de Administración de una Universidad privada de Lima respecto de su personalidad eficaz.

\section{Hipótesis}

1. Existe una relación significativa entre la personalidad eficaz y las capacidades emprendedoras en los estudiantes de la Facultad de Administración de una Universidad privada de Lima.

2. Existen relaciones significativas entre los diferentes componentes de la personalidad eficaz y las diversas capacidades emprendedoras, en los estudiantes de la Facultad de Administración de una Universidad privada de Lima. 
3. Existen diferencias significativas entre los alumnos varones y mujeres de la Facultad de Administración de una Universidad privada de Lima respecto de su personalidad eficaz.

4. Existen diferencias significativas entre los alumnos varones y mujeres de la Facultad de Administración de una Universidad privada de Lima respecto de sus capacidades emprendedoras.

5. Existen diferencias significativas entre los estudiantes considerando su ciclo académico, de la Facultad de Administración de una Universidad privada de Lima respecto de sus capacidades emprendedoras.

6. Existen diferencias significativas entre los estudiantes considerando su ciclo académico, de la Facultad de Administración de una Universidad privada de Lima respecto de su personalidad eficaz.

\section{MÉTODOS Y RESULTADOS}

\section{Método}

El método de investigación utilizado fue el método descriptivo; ya que éste describe las situaciones y eventos, además mide diversos aspectos del fenómeno a investigar. El estudio descriptivo se desarrolla describiendo las situaciones y eventos, es decir cómo se manifiesta el fenómeno a investigar, ya que éste busca especificar las propiedades importantes del problema en cuestión. El método descriptivo mide independientemente los conceptos y también pueden ofrecer la posibilidad de predicciones aunque sean muy rudimentarias. (Sánchez y Reyes, 2002).

\section{Diseño de investigación}

Para la selección del diseño de investigación se ha utilizado como base el libro de Hernández, Fernández y Baptista (2010) titulado "Metodología de la Investigación" Según estos autores el diseño adecuado para esta investigación es de tipo descriptivo correlacional.

$\mathrm{Su}$ diagrama representativo es el siguiente:

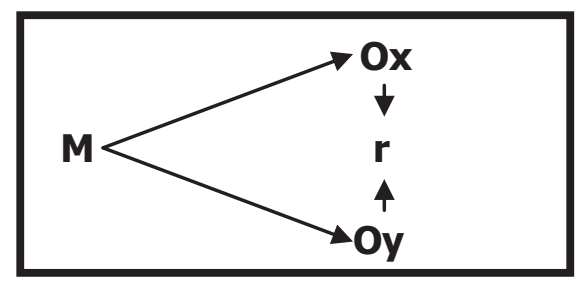

En el esquema: 
CAPACIDADES EMPRENDEDORAS Y PERSONALIDAD EFICAZ EN ESTUDIANTES DE UNA UNIVERSIDAD PRIVADA DE LIMA

$$
\begin{array}{ll}
\mathbf{M} & =\text { Muestra de investigación } \\
\mathbf{O x}, \mathbf{O y} & =\text { Observaciones de las variables } \\
\mathbf{r} & =\text { Relaciones entre variables }
\end{array}
$$

En este caso, se quiere correlacionar las variables personalidad eficaz y capacidades emprendedoras en los estudiantes de la Facultad de Administración de una Universidad privada de Lima.

\section{Muestra}

La muestra de estudio que utilizamos, en tanto se ajusta a nuestras necesidades, es una muestra no probabilística de tipo intencionado en la medida que es el investigador quien a determinado de manera intencional el lugar y la muestra con la que se trabajo. En el presente caso la muestra estuvo constituida por el total de los alumnos de la Facultad de Administración de una Universidad privada de Lima, tal como se presenta a continuación:

Tabla $\mathrm{N}^{\circ}$ 1. Composición general de la muestra

\begin{tabular}{lcc}
\hline Sexo & F & $\%$ \\
\hline Masculino & 55 & 35,5 \\
Femenino & 100 & 64,5 \\
\hline Ciclo & & \\
\hline I & 26 & 16,8 \\
II & 32 & 20,6 \\
III & 24 & 15,5 \\
IV & 40 & 25,8 \\
V & 14 & 9,0 \\
VI & 19 & 12,3 \\
\hline
\end{tabular}

\section{Instrumentos}

Los instrumentos utilizados en el desarrollo de la presente investigación, fue el Inventario de Personalidad Eficaz elaborado por Martín del Buey y compuesto por siete sub escalas: Autoconcepto, Atribuciones académicas, Capacidad resolutiva, Autoestima, Expectativa de éxito, Habilidades sociales y Afrontamiento de problemas.

Igualmente se utilizo el Inventario de Capacidades Emprendedoras de Moriano, J. y compuesto por ocho sub escalas: Riesgo e incertidumbre, Creatividad e innovación, Organización y planificación, Comunicación, Liderazgo, Redes sociales, Detección de oportunidades y Gestión Innovadora.

En ambos casos los resultados son calificados sobre la base de una escala Likert 
de cinco puntos, los cuales permitían expresar en términos de frecuencia la ocurrencia de los aspectos indicados y fluctúan desde Muy en desacuerdo (1) para el menor valor, hasta Muy de Acuerdo (5) para la mayor valoración.

\section{Análisis de validez y confiabilidad de los instrumentos}

En la medida de que se necesita garantizar la idoneidad de los instrumentos de evaluación, se procedió a realizar los análisis estadísticos respectivos que nos indiquen sus niveles de validez y confiabilidad. El análisis psicométrico del Inventario de Personalidad Eficaz efectuado con el coeficiente alfa de cronbach alcanza un valor de 0.92 , lo cual indica que el instrumento es confiable.

El análisis psicométrico del Inventario de Capacidades efectuado con el coeficiente alfa de cronbach alcanza un valor de 0.92 , lo cual indica que el instrumento es confiable.

Los análisis de la Validez de los instrumentos realizados a través del Análisis factorial Exploratorio nos muestran que las pruebas presentan validez de Constructo.

\section{Resultados}

Tabla $\mathrm{N}^{\mathrm{o}} 2$

Análisis de la Bondad de Ajuste de la Curva Normal de las Variables estudiadas

\begin{tabular}{lcccc}
\hline Item & M & D. E. & K-SZ & Sig. \\
Autoconcepto & 29,16 & 3,16 & 1,02 &, 243 \\
Atribuciones académicas & 28,33 & 3,53 & 1,17 &, 126 \\
Capacidad resolutiva & 20,41 & 2,46 & 1,26 &, 082 \\
Autoestima & 21,69 & 2,50 & 1,22 &, 084 \\
Expectativa de éxito & 29,46 & 3,48 & 1,30 &, 065 \\
Habilidades sociales & 28,99 & 3,29 & 1,28 &, 067 \\
Afrontamiento de problemas & 28,90 & 3,22 & 1,11 &, 165 \\
Capacidad de Riesgo & & & & \\
Capacidad creativa & 22,62 & 4,66 & 1,00 &, 261 \\
Capacidad de Organización & 31,70 & 4,92 & 1,10 &, 171 \\
Capacidad de comunicación & 37,26 & 6,29 & 1,05 &, 218 \\
Capacidad de Liderazgo & 38,83 & 6,36 & 0,62 &, 825 \\
Capacidad de crear redes sociales & 39,72 & 5,96 & 0,82 &, 501 \\
Capacidad de detección de & 15,98 & 2,57 & 1,31 &, 062 \\
oportunidades & 19,50 & 3,38 & 1,30 &, 058 \\
Capacidad de trabajo en equipo & 30,87 & 5,08 & 0,79 &, 559 \\
\hline
\end{tabular}

$\mathrm{n}=\mathbf{1 5 5}$ 
Los resultados del análisis exploratorio de los datos (ver tabla $\mathrm{N}^{\circ} 2$ ), en lo que se refiere a la forma de distribución, efectuado a través del test de bondad de ajuste a la curva normal de Kolmogorov-Smirnov, indica que en todas las variables estudiadas, se obtienen coeficientes K-S Z que no son estadísticamente significativos, por lo que se puede concluir que no se presentan adecuadas aproximaciones a la curva normal, por lo que es factible utilizar para el análisis de los datos, estadísticas paramétricas.

Tabla $\mathrm{N}^{\mathrm{o}} 3$

Análisis de las Correlaciones ( $\mathrm{r}$ de Pearson) entre Personalidad eficaz y Capacidades emprendedoras

\begin{tabular}{cc} 
Variables & Capacidades emprendedoras \\
Personalidad eficaz & 0,68 \\
\hline$* \mathbf{p}<. \mathbf{0 5} * * \mathbf{p}<. \mathbf{0 1} * * * \mathbf{p}<\mathbf{. 0 0 1}$ & $\mathbf{n}=\mathbf{1 5 5}$
\end{tabular}

El análisis de las correlaciones entre la ética profesional y el desempeño docente presentados en la Tabla $\mathrm{N}^{0} 3$, indica que existen correlaciones significativas y positivas entre los totales de las variables Personalidad eficaz y Capacidades emprendedoras $(\mathrm{r}=0,68)$.

Tabla $\mathrm{N}^{\circ} 4$

Análisis de Correlación entre las dimensiones de la Personalidad eficaz y las dimensiones de las Capacidades emprendedoras

\begin{tabular}{lcccccccc}
\hline Variables & Riesgo & Cretiv & Organiz & Comuni & Lider & Redes & Dtecc & Equipo \\
Autoconcepto & $0,30^{* *}$ & $0,45^{* *}$ & $0,50^{* *}$ & $0,50^{* *}$ & $0,56^{* *}$ & $0,44^{* *}$ & $0,47^{* *}$ & $0,53^{* *}$ \\
$\begin{array}{l}\text { Atribuciones } \\
\text { académicas }\end{array}$ & $0,20^{*}$ & $0,44^{* *}$ & $0,50^{* *}$ & $0,37^{* *}$ & $0,47^{* *}$ & $0,33^{* *}$ & $0,39^{* *}$ & $0,44^{* *}$ \\
Capacidad resolutiva & $0,27^{* *}$ & $0,38^{* *}$ & $0,44^{* *}$ & $0,35^{* *}$ & $0,49^{* *}$ & $0,40^{* *}$ & $0,39^{* *}$ & $0,44^{* *}$ \\
Autoestima & $0,29^{* *}$ & $0,45^{* *}$ & $0,47^{* *}$ & $0,43^{* *}$ & $0,49^{* *}$ & $0,37^{* *}$ & $0,41^{* *}$ & $0,46^{* *}$ \\
Expectativa de éxito & $0,31^{* *}$ & $0,49^{* *}$ & $0,51^{* *}$ & $0,48^{* *}$ & $0,54^{* *}$ & $0,41^{* *}$ & $0,45^{* *}$ & $0,49^{* *}$ \\
Habilidades sociales & $0,35^{* *}$ & $0,55^{* *}$ & $0,56^{* *}$ & $0,55^{* *}$ & $0,58^{* *}$ & $0,47^{* *}$ & $0,50^{* *}$ & $0,60^{* *}$ \\
$\begin{array}{l}\text { Afrontamiento de } \\
\text { problemas }\end{array}$ & $0,40^{* *}$ & $0,54^{* *}$ & $0,58^{* *}$ & $0,59^{* *}$ & $0,61^{* *}$ & $0,46^{* *}$ & $0,55^{* *}$ & $0,58^{* *}$ \\
\hline * & & & & & & & & \\
\hline
\end{tabular}

$* \mathbf{p}<.05 * * \mathbf{p}<.01 * * * \mathbf{p}<.001 \quad \mathrm{~N}=155$ 
Los resultados presentados en la Tabla $\mathrm{N}^{0} 4$ nos indican que existen correlaciones significativas y positivas en todos los casos.

Tabla $\mathrm{N}^{\mathrm{o}} 5$

Prueba $\mathrm{Z}$ de comparación de medias de los puntajes de las Escalas de Personalidad Eficaz por Sexo

\begin{tabular}{lcccccc}
\hline \multicolumn{1}{c}{ Variables } & \multicolumn{2}{c}{ Varón } & \multicolumn{2}{c}{ Mujer } \\
& \multicolumn{2}{c}{ N=55 } & \multicolumn{2}{c}{ N=100 } & Z & Sig. \\
& M & D.E. & M & D.E & & \\
Autoconcepto & 28,94 & 2,91 & 29,29 & 3,30 & $-0,64$ &, 519 \\
Atribuciones académicas & 28,94 & 3,08 & 28,00 & 3,73 & 1,60 &, 112 \\
Capacidad resolutiva & 20,34 & 2,51 & 20,46 & 2,44 & $-0,27$ &, 783 \\
Autoestima & 22,09 & 2,03 & 21,48 & 2,72 & 1,45 &, 147 \\
Expectativa de éxito & 30,10 & 2,68 & 29,11 & 3,82 & 1,71 &, 088 \\
Habilidades sociales & 29,58 & 3,06 & 28,67 & 3,39 & 1,65 &, 100 \\
Afrontamiento de problemas & 29,01 & 2,89 & 28,85 & 3,40 & 0,30 &, 757 \\
Total PE & 189,03 & 15,64 & 185,86 & 19,19 & 1,05 &, 295 \\
\hline
\end{tabular}

$* \mathbf{p}<.05$

$\mathbf{N}=\mathbf{1 5 5}$

El análisis comparativo de las Escalas de Personalidad Eficaz por sexo (ver Tabla $\mathrm{N}^{\mathrm{o}}$ 5), indica que no existen diferencias estadísticas significativas en caso alguno, por lo que se debe señalar que tanto los hombres como las mujeres presentan valores similares en cada una de las sub escalas y en el total de la prueba.

Tabla $\mathrm{N}^{0} 6$

Prueba $\mathrm{Z}$ de comparación de medias de los puntajes de las Escalas de las capacidades emprendedoras por Sexo

\begin{tabular}{lcccccc}
\hline \multirow{2}{*}{ Variables } & \multicolumn{2}{c}{ Varón } & \multicolumn{2}{c}{ Mujer } \\
& $\mathrm{N}=55$ & $\mathrm{D} . \mathrm{E}$. & $\mathrm{M}$ & $\mathrm{D} . \mathrm{E}$ & & Sig. \\
Riesgo & 23,20 & 5,02 & 22,31 & 4,45 & 1,13 &, 257 \\
creatividad & 32,00 & 4,87 & 31,54 & 4,97 & 0,55 &, 580 \\
Organización & 37,16 & 6,01 & 37,32 & 6,47 & $-0,14$ &, 883 \\
Comunicación & 38,49 & 5,55 & 39,03 & 6,79 & $-0,50$ &, 616 \\
Liderazgo & 39,67 & 5,46 & 39,75 & 6,25 & $-0,07$ &, 939 \\
Redes sociales & 15,81 & 2,68 & 16,08 & 2,51 & $-0,60$ &, 546 \\
Detección de & 19,36 & 3,31 & 19,58 & 3,43 & $-0,38$ &, 705 \\
oportunidades & 31,34 & 4,40 & 30,62 & 5,42 & 0,84 &, 397 \\
Trabajo en equipo & 237,05 & 29,67 & 236,23 & 33,92 & 0,15 &, 880 \\
Total CE & & & & & & \\
\hline
\end{tabular}

$* p<.05 \quad N=155$ 
El análisis comparativo de las Escalas de las Capacidades emprendedoras por sexo (ver Tabla $\mathrm{N}^{\circ} 6$ ), indica que no existen diferencias estadísticas significativas en ningún caso, por lo que podemos afirmar que tanto los varones como las mujeres poseen el mismo nivel de capacidades emprendedoras.

Tabla $\mathrm{N}^{\circ} 7$

Análisis de Varianza de un Factor de la Personalidad eficaz por ciclo de estudio

\begin{tabular}{lccccccccc}
\hline & I ciclo & II ciclo & III ciclo & IV ciclo & V ciclo & VI ciclo & & \\
Variables & $\mathbf{N}=\mathbf{2 6}$ & $\mathbf{N}=\mathbf{3 2}$ & $\mathbf{N}=\mathbf{2 4}$ & $\mathbf{N}=\mathbf{4 0}$ & $\mathbf{N}=\mathbf{1 4}$ & $\mathbf{N = 1 9}$ & $\mathbf{F}$ & Sig. \\
& $\mathbf{M}$ & $\mathbf{M}$ & $\mathbf{M}$ & $\mathbf{M}$ & $\mathbf{M}$ & $\mathbf{M}$ & & \\
Autoconcepto & 29,42 & 29,50 & 29,75 & 27,97 & 28,71 & 30,36 & 2,07 &, 071 \\
Atribuciones & 28,76 & 28,15 & 28,91 & 26,67 & 29,14 & 30,21 & 3,45 &, $006^{* *}$ \\
Capacidad resolutiva & 20,07 & 20,59 & 20,70 & 19,87 & 20,71 & 21,15 & 0,97 &, 438 \\
Autoestima & 21,53 & 21,78 & 22,25 & 20,97 & 21,85 & 22,47 & 1,31 &, 261 \\
Expectativa de éxito & 29,84 & 29,37 & 30,58 & 27,77 & 30,14 & 30,73 & 3,27 &, $008^{* *}$ \\
HHSS & 28,80 & 29,06 & 30,12 & 28,15 & 29,14 & 29,36 & 1,16 &, 328 \\
Afrontamiento & 28,65 & 29,18 & 30,29 & 27,62 & 29,07 & 29,63 & 2,54 &, $030^{*}$ \\
Total PE & 187,11 & 187,65 & 192,62 & 179,05 & 188,78 & 193,94 & 2,77 &, $020^{*}$ \\
\hline
\end{tabular}

$* \mathbf{p}<.05 * * \mathbf{p}<.01 * * * \mathbf{p}<.001 \quad \mathrm{~N}=155$

Diferencias significativas por pares de medias a través del test de Scheffe: a, b, c

Los resultados del análisis de varianza de un factor de las áreas de la Personalidad eficaz por ciclo de estudio indican que existen diferencias estadísticas significativas en los siguientes aspectos:

a. Atribuciones Académicas $(\mathrm{F}=3,45 \mathrm{p}<.01)$ notándose que los alumnos del VI ciclo $(\mathrm{M}=30,21)$ y del $\mathrm{V}$ ciclo $(\mathrm{M}=29,14)$ superan a los alumnos de los demás ciclos académicos.

b. Expectativas de éxito $(\mathrm{F}=3,27 \mathrm{p}<.01)$ notándose que los alumnos del VI ciclo $(\mathrm{M}=30,73)$ y del III ciclo $(\mathrm{M}=30,58)$ superan a los alumnos de los demás ciclos académicos.

c. Afrontamiento de Problemas $(\mathrm{F}=2,54 \mathrm{p}<.05)$ notándose que los alumnos del III ciclo $(\mathrm{M}=30,29)$ y del VI ciclo $(\mathrm{M}=29,63)$ superan a los alumnos de los demás ciclos académicos.

d. Total Personalidad Eficaz $(\mathrm{F}=2,77 \mathrm{p}<.05)$ notándose que los alumnos del VI ciclo $(M=193,94)$ y del III ciclo $(M=192,62)$ superan a los alumnos de los demás ciclos académicos. 
Manuel Torres V., Manuel Torres L.

Tabla $\mathrm{N}^{\circ} 8$

Análisis de Varianza de un Factor de las capacidades emprendedoras por ciclo de estudio

\begin{tabular}{lllllllll}
\hline & I ciclo & II ciclo & III ciclo & IV ciclo & V ciclo & VI ciclo & & \\
Variables & $\mathbf{N}=\mathbf{2 6}$ & $\mathbf{N}=\mathbf{3 2}$ & $\mathbf{N}=\mathbf{2 4}$ & $\mathbf{N}=\mathbf{4 0}$ & $\mathbf{N}=\mathbf{1 4}$ & $\mathbf{N}=\mathbf{1 9}$ & $\mathbf{F}$ & Sig. \\
& $\mathbf{M}$ & $\mathbf{M}$ & $\mathbf{M}$ & $\mathbf{M}$ & $\mathbf{M}$ & $\mathbf{M}$ & & \\
Riesgo & 22,57 & 23,31 & 22,41 & 22,65 & 22,00 & 22,21 & 0,22 &, 952 \\
creatividad & 31,73 & 31,78 & 33,37 & 30,77 & 30,57 & 32,21 & 1,02 &, 404 \\
Organización & 38,03 & 37,18 & 37,50 & 35,62 & 37,64 & 39,21 & 1,00 &, 419 \\
Comunicación & 40,00 & 38,09 & 39,29 & 37,82 & 37,50 & 41,05 & 1,07 &, 377 \\
Liderazgo & 40,00 & 40,62 & 40,12 & 37,92 & 38,64 & 41,89 & 1,52 &, 185 \\
Redes sociales & 15,84 & 16,31 & 15,62 & 16,00 & 15,71 & 16,26 & 0,28 &, 922 \\
Detección de oportunidades & 19,61 & 20,03 & 19,33 & 19,05 & 19,00 & 20,00 & 0,45 &, 811 \\
Trabajo en equipo & 31,69 & 31,25 & 31,41 & 29,85 & 30,78 & 30,68 & 0,54 &, 741 \\
Total CE & 239,50 & 238,59 & 239,08 & 229,70 & 231,85 & 243,52 & 0,68 &, 636 \\
\hline
\end{tabular}

$* \mathbf{p}<.05 * * \mathbf{p}<.01 * * * \mathbf{p}<.001 \quad \mathbf{N}=155$

Diferencias significativas por pares de medias a través del test de Scheffe: a, b, c

Los resultados del análisis de varianza de un factor de las áreas de las capacidades emprendedoras por ciclo de estudio indican que no existen diferencias estadísticas significativas en caso alguno, lo que implica que los estudiantes, sin importar su ciclo académico, presentan los mismos niveles de capacidades emprendedoras.

\section{DISCUSIÓN}

Los resultados del análisis psicométrico de la prueba de Personalidad eficaz nos muestran que todos los ítems forman parte de dicho inventario, y corresponden a cada una de las sub escalas asignadas en el inventario. El instrumento es confiable en la medida que obtuvo un coeficiente Alfa de Cronbach de 0,92.

En cuanto a la validez, el resultado del Análisis Factorial Exploratorio indica que la prueba está conformada por un solo factor que en general explica el 68,70\% de la varianza total. Por otra parte observamos que la medida de adecuación del muestreo de Kaiser-Meyer-Olkin alcanza un valor de 0.90 que puede considerarse como adecuado, mientras que el test de esfericidad de Bartlett presenta un valor que es significativo. Estos hallazgos permiten concluir que la prueba de personalidad eficaz presenta validez de constructo.

En cuanto a la escala de capacidades emprendedoras, según los resultados del análisis de ítems, los 62 reactivos deben permanecer conformando el Cuestionario en las escalas asignadas por los autores de la versión original. Asimismo, el coeficiente Alfa de Cronbach alcanzado es de 0.92 , lo cual indica que la escala es confiable.

Los resultados del Análisis Factorial Exploratorio de la prueba de capacidades emprendedoras indican que está conformada por un solo factor, que explica el $77,46 \%$ de la varianza total. Por otra parte tenemos que la medida de adecuación 
del muestreo de Kaiser-Meyer-Olkin alcanza un valor de 0.93 que puede considerarse como adecuado, mientras que el test de esfericidad de Bartlett presenta un valor que es significativo. Estos hallazgos permiten concluir que la prueba de capacidades emprendedoras presenta validez de constructo.

De acuerdo a estos resultados las pruebas son válidas y confiables y por lo tanto se pueden usar en el desarrollo del presente trabajo y de cualquier otro que se quiera realizar utilizando estas variables. Esto es sin duda un primer aporte de este trabajo que seguramente va a ser adecuadamente aprovechado por otros investigadores interesados en profundizar el estudio sobre el emprendedorismo y la personalidad eficaz.

Al revisar los resultados obtenidos podemos afirmar que la hipótesis principal de investigación "Existe una relación significativa entre la personalidad eficaz y las capacidades emprendedoras en los estudiantes de la Facultad de Administración de una Universidad privada de Lima", ha sido aceptada, esto es, se ha comprobado que existen correlaciones positivas y significativas entre estas variables, por lo que hoy resulta de vital importancia fomentar las capacidades emprendedoras y la personalidad eficaz en los estudiantes universitarios.

Los resultados obtenidos nos permiten reafirmar las propuestas de Dolabela (2005) cuando señala que "ser emprendedor no es solo una cuestión de acumulación de conocimientos, sino la interiorización de valores, actitudes, comportamientos, formas de percepción del mundo y de sí mismo volcados a actividades en que el riesgo, la capacidad de innovar, perseverar y de convivir con la incertidumbre son elementos indispensables en esta era", por lo que la formación y capacitación de la personalidad eficaz y las capacidades emprendedoras devienen en una necesidad urgente de la universidad; y es que la formación en capacidades emprendedoras se ha tornado en una necesidad imperiosa para garantizar y avanzar en la sociedad del bienestar.

Tenemos que considerar que el emprendedor es un transformador de primera magnitud que contribuye a la producción de nuevas ofertas y oportunidades para su comunidad. Necesitamos de los emprendedores para seguir avanzando en la construcción de una sociedad genuina, rica y solidaria.

La sociedad necesita emprendedores y la Universidad tiene que proporcionárselos. Los diversos estudios muestran la necesidad de profundizar en el comportamiento y el papel de las universidades ante el reto de formar emprendedores, así por ejemplo, Olmos y Sastre (2007), con formación en las ciencias económicas y de administración, presentan a la comunidad científica un cuestionamiento sobre la responsabilidad de las universidades en la estimulación organizada hacia sus estudiantes en la creación de empresas y si cumplen o no este propósito. Sus resultados textuales dicen "la actitud emprendedora declina en los estudiantes desde los primeros años de sus estudios hasta que llega el momento de su incorporación al mercado laboral. Todo parece indicar que los conocimientos aportados incrementan sus competencias de gestión pero les orientan hacia salidas laborales más conservadoras, como son el trabajo por cuenta ajena, y no les motivan hacia el 
autoempleo." Olmos y Sastre (2007) sostienen que puede existir emprendimiento en las universidades, pero depende del apoyo de las mismas.

El reto es posible en condiciones normales, y más aún, como es nuestro caso, con la generación de personas mejor preparadas de la historia, momento oportuno para generar los mecanismos para que los jóvenes se formen como emprendedores y puedan diseñar nuevas y atractivas fórmulas de negocios y empleo.

Pero en este proceso es imprescindible que se forme también una personalidad eficaz en estos jóvenes. Personalidad eficaz y emprendimiento son dos fenómenos íntimamente unidos, es imposible imaginar una formación en personalidad eficaz sin los fundamentos emprendedores ni viceversa. Como características principales de la personalidad eficaz podemos destacar que se aprende, dicho como ejemplo, si a una personalidad eficaz lo identificamos por diez facetas prácticas de su comportamiento, se trataría pues de sistematizarlas para que la gente pueda adquirirlas a través de la formación, de la misma forma que para formar a un escritor hay que instruirle en la literatura, la lengua y sus recursos, la lectura sistemática, la escritura diaria y su perfeccionamiento paulatino, este es el camino que debemos seguir para formar personalidades eficaces y emprendedoras.

\section{CONCLUSIONES}

1. Los análisis estadísticos realizados demuestran que la personalidad eficaz se encuentra relacionada significativamente con las capacidades emprendedoras $(\mathrm{r}=0,68)$.

2. Los resultados de los análisis estadísticos realizados demuestran que los diferentes componentes de la personalidad eficaz se encuentran relacionados a las diversas capacidades emprendedoras en los estudiantes de de la Facultad de Administración de una Universidad privada de Lima.

3. Los análisis estadísticos demuestran que no existen diferencias estadísticas significativas en caso alguno, por lo que se debe señalar que tanto los hombres como las mujeres presentan valores similares en cada una de las sub escalas de la personalidad eficaz.

4. Los resultados obtenidos indican que no existen diferencias estadísticas significativas en ningún caso, por lo que podemos afirmar que tanto los varones como las mujeres poseen el mismo nivel de capacidades emprendedoras.

5. Los resultados del análisis de varianza de las áreas de la Personalidad eficaz por ciclo de estudio indican que existen diferencias estadísticas significativas en los casos de Atribuciones Académicas, Expectativas de éxito, Afrontamiento de Problemas y en el Total de la Personalidad Eficaz.

6. Los resultados del análisis de varianza de las áreas de las capacidades emprendedoras por ciclo de estudio indican que no existen diferencias estadísticas significativas en caso alguno, lo que implica que los estudiantes, sin importar su ciclo académico, presentan los mismos niveles de capacidades emprendedoras. 
CAPACIDADES EMPRENDEDORAS Y PERSONALIDAD EFICAZ EN ESTUDIANTES DE UNA UNIVERSIDAD PRIVADA DE LIMA

\section{REFERENCIAS BIBLIOGRÁFICAS}

Alcaraz Rodríguez, R. (2001) El emprendedor de éxito: guía de negocios. Mc Graw Hill, México.

Bermejo, M. (2003) Crea tu propia empresa: estrategias para su puesta en marcha y supervivencia. Mc Graw Hill, España.

Cano, C.J.; García, J.; Gea, A.B. (2003). Actitudes emprendedoras y creación de empresas en los estudiantes universitarios. Almería: Servicio de Publicaciones de la Universidad de Almería y Consejo Social.

Comisión Europea (2003). Libro verde. El espíritu empresarial en Europa. Bruselas: Dirección General de Empresa.

Delors, (1996): La educación encierra un tesoro. Informe sobre la educación para el siglo XXI. Paris: UNESCO.

Díaz Mérigo, A. (1982). El Dirigente. (2 . Ed.) México: McGraw-Hill.

Dolabela F. (2003). Pedagogía Emprendedora. Editora de Cultura, Sao Paulo.

Fernández Aguado, J. (2000) 1000 consejos para un emprendedor. Inversiones editoriales dossat 200, España.

Garzón Castrillon M. (1995). El modelo intrapreneuring (intraemprendedor), en revista Administrate Hoy, No. 12 del 5 de Abril. México.

Gallart, M. A. y C. J. (1995) Competencias laborales: tema clave en la articulación educación-trabajo, Boletín Educación y Trabajo, 6(2). Buenos Aires: Red Latinoamericana de Educación y Trabajo CIID-CENEP, diciembre.

González, F.J. (2004) Incidencia del Marco Institucional en la Capacidad Emprendedora de los Jóvenes Empresarios de Andalucía. Tesis doctoral Universidad de Sevilla. España.

Hernández, R. Fernández, C. y Baptista, P. (2010). Metodología de la investigación. México: Mc Graw Hill

Ibarra, E. (2000). Los primeros pasos al mundo empresarial. México: Prentice Hall.

Kantis, H. (2001) Desarrollo emprendedor: América latina y la experiencia internacional. Nomos, Colombia.

Liñán, F. (2004) Educación empresarial y modelo de intenciones. Formación para un empresariado de calidad. 2004. Tesis (Doctorado en Economía Aplicada) Departamento Economía Aplicada I, Universidad de Sevilla, Sevilla.

Martín del Buey, F. (1997). Programa integrado de acción tutorial. Valparaíso: Revista de Orientación Educacional, 19-20, 71-88 Universidad de Playa Ancha. 
Martín del Buey, F. y Fernández Zapico, A. (2003). Programa de desarrollo de la personalidad eficaz en contextos educativos: primeros resultados. Magister, 19, 277291. Oviedo: Universidad de Oviedo.

Martín del Buey, F., Martín Palacio, E, Fernández Zapico, A., Dapelo Pellerano, B. y Marcone Trigo, R. (2004). Evaluación de la personalidad eficaz en contextos educativos: primeros resultados. Chile: Revista de Orientación Educacional, 33-34, 79-101.

Mc Clelland, D. C. (1961): La sociedad ambiciosa, Ediciones Guadarrama, Madrid.

Moriano, J. A.; Sánchez, M. L.; Palací, F.J. (2004): Un estudio descriptivo sobre los emprendedores en España, la República Checa y Bulgaria, en Roig, S.; Ribeiro, D.; Torcal, R.; De la Torre, A.; Cerver, E. (2004): "El emprendedor innovador y la creación de empresas de $I+D+I$ " Servei de Publicacións Universitat de Valencia, cap.10, pp. 161-178.

Olivares, S. (1993). Comportamiento Organizacional. México: Limusa.

Olmos, R. y Sastre, M. (2007) La Actitud Emprendedora durante la vida académica de los estudiantes Universitarios. Madrid. Universidad Complutense de Madrid.. Cuadernos de Estudios Empresariales. Vol.17 p. 95 - 116.

Padua, J. (1997) Como evaluar las acciones de capacitación. Argentina: Vergara.

Rubio, E.A.; Cordón, E. y Agote, L.A. (1999). Actitudes hacia la Creación de Empresas: un Modelo Explicativo. Revista Europea de Dirección y Economía de la Empresa, 8

Shefsky, Lloyd E (1998) Los emprendedores no nacen se hacen: aprenda los secretos de 200 emprendedores exitosos. Mc Graw Hill, España

Sánchez, H y Reyes, C. (2002). Metodología y Diseños en la Investigación Científica. Lima: URP. Editorial Universitaria.

Stoner, J. y Wankel, Ch. (2006). Administración Tercera edición. Editorial Prentice-Hall. Hispanoamericana S.A. México.

Vainrub, R. (2004) Convertir sueños en realidades: una guía para emprendedores. Ediciones IESA, Caracas. 